\title{
Attenuation compensation in least-squares reverse time migration using the visco-acoustic wave equa- tion
}

Gaurav Dutta*, Kai Lu, Xin Wang and Gerard T. Schuster, King Abdullah University of Science and Technology

\section{SUMMARY}

Attenuation leads to distortion of amplitude and phase of seismic waves propagating inside the earth. Conventional acoustic and least-squares reverse time migration do not account for this distortion which leads to defocusing of migration images in highly attenuative geological environments. To account for this distortion, we propose to use the visco-acoustic wave equation for least-squares reverse time migration. $\mathrm{Nu}-$ merical tests on synthetic data show that least-squares reverse time migration with the visco-acoustic wave equation corrects for this distortion and produces images with better balanced amplitudes compared to the conventional approach.

\section{INTRODUCTION}

Fluids trapped in overburden structures cause strong attenuation of $\mathrm{P}$-waves which hamper the resolution of migrated images. This can be attributed to the fact that the real earth is anelastic and this causes distortion of the amplitude and phase of the propagating seismic waves (Aki and Richards (1980)). If the attenuation is too strong, ignoring it during migration can lead to incorrect positioning of reflectors below these layers. Attenuation of P-waves can be quantified by an attenuation parameter or a quality factor, $Q_{P}$, which accounts for the amplitude attenuation and the phase shift as a function of the the frequency content of the propagating waves and the distance travelled. Lower values of $Q_{P}$ imply more energy loss of the wave per cycle or very high attenuation. The values of $Q_{P}$ for rocks like gas-sandstone and shale are very low $\left(Q_{P}\right.$ $\approx 15-30$ ) and this necessitates the need to account for $Q_{P}$ during migration for more accurate and better resolved images.

Reverse time migration (RTM) has become the standard migration algorithm for imaging in complex geological settings like the Gulf of Mexico. Conventional acoustic RTM uses the two-way wave equation for computing the Green's functions (Baysal et al. (1983), McMechan (1983)) and is more accurate than the integral based Kirchhoff methods. Dai et al. (2012) extended the idea of least-squares migration (Nemeth et al. (1999)) to least-squares reverse time migration (LSRTM) and showed that LSRTM can mitigate the artifacts of RTM and can produce images of better resolution compared to standard RTM. However, RTM and LSRTM do not take into account the attenuation due to $Q_{P}$ if the standard acoustic wave equation is used for wavefield extrapolation. In this work, we propose to use the visco-acoustic wave equation instead of the standard acoustic wave equation for LSRTM and show with numerical tests that LSRTM using the visco-acoustic wave equation produces images with better balanced amplitudes and accurate positioning of reflectors compared to acoustic LSRTM when the subsurface attenuation is very strong.

\section{LEAST-SQUARES REVERSE TIME MIGRATION WITH THE VISCO-ACOUSTIC WAVE EQUATION}

The stress-strain relation for a visco-acoustic medium is given by (Christensen (1982), Carcione et al. (1988)),

$$
\sum_{k=0}^{m} c_{k} \frac{d^{k} p}{d t^{k}}=\sum_{k=0}^{m} d_{k} \frac{d^{k} e}{d t^{k}}
$$

where $p$ denotes the pressure field, $e$ denotes the trace of the strain tensor matrix, $d^{k} / d t^{k}$ represents the $k$-th order time derivative and $c_{k}$ and $d_{k}$ are coefficients related to the material properties of the medium. The pressure field can be expressed explicitly from equation (1) using Laplace transform methods as,

$p(t)=-M_{R} \int_{\infty}^{t} \dot{e}(\tau)\left[1-\sum_{l=1}^{L}\left(1-\frac{\tau_{\varepsilon l}}{\tau_{\sigma l}}\right) \exp \left(-\frac{t-\tau}{\tau_{\sigma l}}\right)\right] d \tau$,

where $\tau_{\sigma l}$ and $\tau_{\varepsilon l}$ denote material relaxation times for the $l$ th mechanism, $L$ is the number of relaxation mechanisms and $M_{R}$ is the relaxed modulus of the medium. The equation of momentum conservation can be written as (Carcione et al. (1988)),

$$
-\frac{\partial}{\partial x_{i}}\left(\frac{1}{\rho} \frac{\partial p}{\partial x_{i}}\right)=\ddot{e}+\frac{\partial}{\partial x_{i}}\left(\frac{1}{\rho} f_{i}\right)
$$

where $\rho$ and $f_{i}$ represent the density and body forces, respectively. Equations (2) and (3) together describe the deformation in a visco-acoustic medium. For numerical modeling, the convolution term in equation (2) is simplified by introducing a memory variable term, $r_{p}$, (Robertsson et al. (1994)) and only one relaxation mechanism $(L=1)$ is sufficient for practical purposes (Blanch et al. (1995)). Thus, for a 2D visco-acoustic medium, the linearized equations of motion and deformation become (Thorbecke and Draganov (2011)),

$$
\begin{aligned}
& \frac{\partial V_{x}}{\partial t}=\frac{1}{\rho} \frac{\partial P}{\partial x}, \\
& \frac{\partial V_{z}}{\partial t}=\frac{1}{\rho} \frac{\partial P}{\partial z} \\
& \frac{\partial P}{\partial t}=\frac{1}{\kappa} \frac{\tau_{\varepsilon}}{\tau_{\sigma}}\left(\frac{\partial V_{x}}{\partial x}+\frac{\partial V_{z}}{\partial z}\right)+r_{p}, \\
& \frac{\partial r_{p}}{\partial t}=-\frac{1}{\tau_{\sigma}}\left(r_{p}+\left(\frac{\tau_{\varepsilon}}{\tau_{\sigma}}-1\right)\left(\frac{1}{\kappa}\right)\left(\frac{\partial V_{x}}{\partial x}+\frac{\partial V_{z}}{\partial z}\right)\right) .
\end{aligned}
$$

Here, $V_{x}$ and $V_{z}$ represent the particle velocities in the $x$ and $z$ directions, respectively, and $\kappa$ represents the bulk modulus of the medium. The relaxation parameters, $\tau_{\sigma}$ and $\tau_{\varepsilon}$, are related to the quality factor, $Q_{P}$, and the central frequency, $f_{w}$, of the source wavelet as (Robertsson et al. (1994)),

$$
\begin{aligned}
\tau_{\sigma} & =\frac{\sqrt{1+\frac{1}{Q_{P}^{2}}-\frac{1}{Q_{P}}}}{f_{w}}, \\
\tau_{\varepsilon} & =\frac{1}{f_{w}^{2} \tau_{\sigma}} .
\end{aligned}
$$




\section{Attenuation LSRTM}

Equation 4 can be used for wavefield extrapolation in a viscoacoustic medium during RTM and LSRTM. Figure 1 shows the effect of attenuation on amplitude and phase for a homogeneous medium with a background velocity of $3000 \mathrm{~m} / \mathrm{s}$ and for different values of $Q_{P}$. The source is excited at the center of the model and the snapshots are taken at $t=0.8 \mathrm{~ms}$. It is evident that lower values of $Q_{P}$ distort the amplitude and phase of the propagating waves.

For visco-acoustic LSRTM, the Born modeling equation can be written as,

$$
P(\mathbf{g}, \mathbf{s}) \approx \int_{\mathbf{x}} G(\mathbf{x} \mid \mathbf{s}) m(\mathbf{x}) G(\mathbf{g} \mid \mathbf{x}) W(\omega) d \mathbf{x},
$$

where $G\left(\mathbf{x}^{\prime} \mid \mathbf{x}\right)$ is the Fourier domain representation of the bandlimited Green's function for a source excited at $\mathbf{x}$ and an observation point at $\mathbf{x}^{\prime}$ and is numerically computed using equation (4). $W(\omega)$ represents the source wavelet for an angular frequency $\omega, m(\mathbf{x})$ represents the reflectivity model and $P(\mathbf{g}, \mathbf{s})$ represents the born-modeled data where $\mathbf{g}$ and $\mathbf{s}$ are the geophone and source coordinates, respectively. For notational convenience, $\omega$ has been dropped from the pressure and the Green's functions terms. The RTM operator can be derived by applying the adjoint operation on equation (6) as,

$$
m(\mathbf{x}) \approx \int_{\omega} \int_{\mathbf{s}} \int_{\mathbf{g}} G(\mathbf{x} \mid \mathbf{s})^{*} P(\mathbf{g}, \mathbf{s}) G(\mathbf{g} \mid \mathbf{x})^{*} d \mathbf{g} d \mathbf{s} d \omega .
$$

Using a matrix-vector notation, the forward modeling operation in equation (6) can be written as,

$$
\mathbf{d}=\mathbf{L} \mathbf{m},
$$

and the adjoint operation or the RTM operation can be written as (Claerbout and Green (2008)),

$$
\mathbf{m} \approx \mathbf{L}^{T} \mathbf{d} .
$$

The reflectivity model, $m(\mathbf{x})$, can be determined using a leastsquares method by minimizing the misfit function, $\varepsilon$, as (Nemeth et al. (1999), Dai et al. (2012)),

$$
\varepsilon=\frac{1}{2}\left\|\mathbf{L m}-\mathbf{d}^{o b s}\right\|^{2} .
$$

Equation (10) can be iteratively solved using any gradient based method like steepest descent as,

$$
\begin{aligned}
& \mathbf{m}^{(i+1)}=\mathbf{m}^{(i)}-\alpha \mathbf{g}^{(i)}, \\
& \mathbf{g}^{(i)}=\mathbf{L}^{T}\left(\mathbf{L m}^{(i)}-\mathbf{d}^{o b s}\right), \\
& \alpha=\frac{\left(\mathbf{g}^{(i)}\right)^{T} \mathbf{g}^{(i)}}{\left(\mathbf{L} \mathbf{g}^{(i)}\right)^{T}\left(\mathbf{L} \mathbf{g}^{(i)}\right)} .
\end{aligned}
$$

Here, $\mathbf{m}^{(i)}$ represents the reflectivity model at the $i$-th iteration, $\mathbf{g}^{(i)}$ and $\alpha$ represent the gradient and the step-length, respectively. The gradient is determined by reverse time migration of the data residuals between the born-modeled data and the observed data which has been calculated using a full finite difference simulation of the visco-acoustic wave equation given in equation (4).

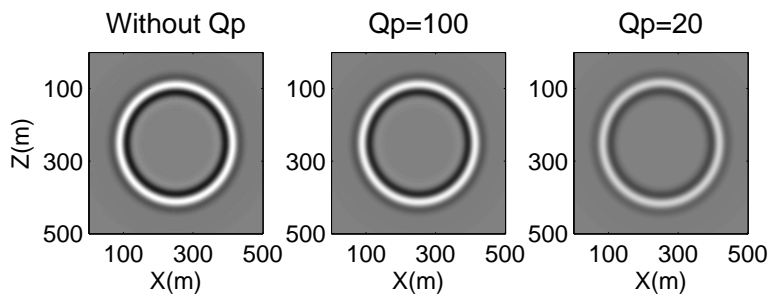

(a)

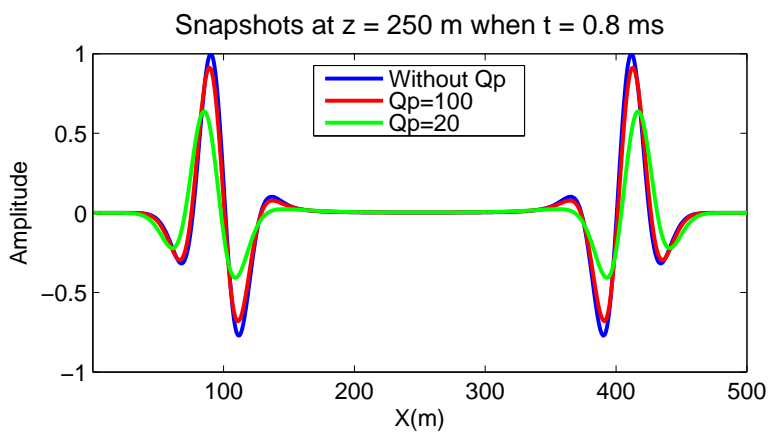

(b)

Figure 1: (a) Wavefield Snapshots at $t=0.8 \mathrm{~ms}$ for a homogeneous medium showing the effect of attenuation for different values of $Q_{P}$. (b) The amplitude and phase distortion due to very low value of $Q_{P}$ can be seen by comparing the three traces.

\section{NUMERICAL EXAMPLES}

The proposed LSRTM algorithm using the visco-acoustic wave equation is tested on a modified Marmousi model shown in Figure 2(a). The $Q_{P}$ distribution, shown in Figure 2(b), is estimated from the true velocity model in Figure 2(a) by assigning the layers with velocities ranging between $2500-3000 \mathrm{~m} / \mathrm{s}$ to have very strong attenuation or very low $Q_{P}$. The migration velocity model, shown in Figure 2(c), is obtained by smoothing the true velocity model. The $Q_{P}$ model for migration, shown in Figure 2(d), is estimated from the migration velocity model using a similar mapping of velocity and $Q_{P}$ values as in the case of the true $Q_{P}$ model.

A 2-8 time-space domain staggered grid finite-difference method is used for visco-acoustic and acoustic RTM and LSRTM in all cases. There are 115 shots fired at a source interval of $40 \mathrm{~m}$ and there are 230 receivers evenly distributed on the surface at an interval of $20 \mathrm{~m}$. A fixed spread acquisition geometry is chosen as all the receivers are used for each source. A Ricker wavelet with a $20 \mathrm{~Hz}$ peak frequency is used as the source wavelet. The observed data having strong attenuation is generated using the velocity and the $Q_{P}$ models shown in Figures 2(a) and (b), respectively. To see the effect of attenuation, the same data is migrated first by taking attenuation into account by using the visco-acoustic wave equation for extrapolation of the source and receiver wavefields and then migrated again by ignoring the effect of attenuation and using the first order acoustic wave equations. Source side illumination is used as the preconditioning factor during LSRTM in both cases. 
Attenuation LSRTM
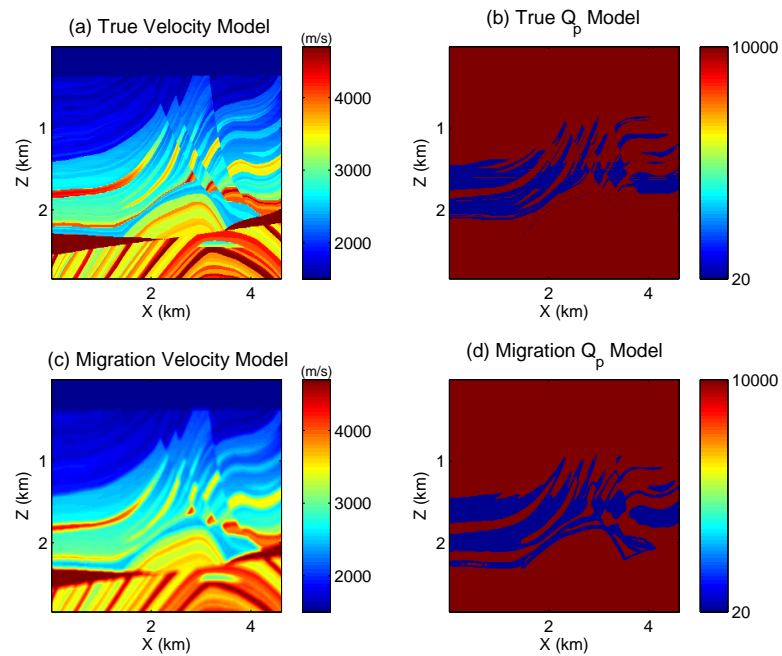

Figure 2: The (a) true velocity model, and (b) the true $Q_{P}$ model used for generating the observed data. (c) Velocity model, and (d) $Q_{P}$ model used for RTM and LSRTM.

Figures 4(a) and 4(c) compare the RTM images when the data having strong attenuation is migrated using the acoustic and the visco-acoustic wave equations, respectively. The LSRTM images after 20 iterations are compared in Figures 4(b) and 4(d) for both the cases. Zoomed sections of the images in Figures 4(b) and 4(d) are shown in Figure 5. It is evident that LSRTM using the acoustic and visco-acoustic wave equations provide similar results in shallow layers where there is very little or no attenuation. However, in deeper layers, where the attenuation effect is very strong, the LSRTM image obtained by using the visco-acoustic wave equation shows better resolution and focusing compared to the LSRTM image obtained using the acoustic wave equation. Also, the migrated amplitudes in the deeper layers are better balanced in the visco-acoustic case. Careful analysis of the images in Figures 5(b) and 5(e) also reveals that the reflectors in the deeper layers are slightly mispositioned when compared to the true reflectivity model. However, for the visco-acoustic LSRTM images in Figures 5(c) and 5(f), all the reflectors are imaged at the right locations. This can be attributed to the fact that strong attenuation not only affects the amplitudes but also the phase of the events and this effect becomes more prominent for the waves that travel large distances and through the high attenuative layers. Migration without taking attenuation because of $Q_{P}$ into consideration cannot correct for this distortion. The convergence curves for acoustic and visco-acoustic LSRTM are also compared in Figure 3. The convergence in this case is better for visco-acoustic LSRTM since the correct physics is accounted for during the modeling and the adjoint operations.

\section{CONCLUSIONS}

We presented a least-squares reverse time migration method using the visco-acoustic wave equation to compensate for the distortion in amplitude and phase of seismic waves propagat- ing in highly attenuative layers. Numerical results using the Marmousi model show that conventional LSRTM using the acoustic wave equation cannot correct for the attenuation loss. However, when the visco-acoustic wave equation is used during LSRTM, the attenuation effect is correctly accounted for. LSRTM using the visco-acoustic wave equation produces images with better balanced amplitudes and accurately positioned reflectors below highly attenuative layers compared to the acoustic LSRTM images. Similar to acoustic LSRTM, visco-acoustic LSRTM is also sensitive to errors in the migration velocity model. Estimation of $Q_{P}$ from real data is also difficult. The $Q_{P}$ tomography methods suggested by Liao and McMechan (1996) and Liao and McMechan (1997) is one possible solution to get a starting $Q_{P}$ model for visco-acoustic LSRTM. More accurate estimation of $Q_{P}$ should be emphasized on, especially during processing, for accurate imaging in gas-sandstones and shales.

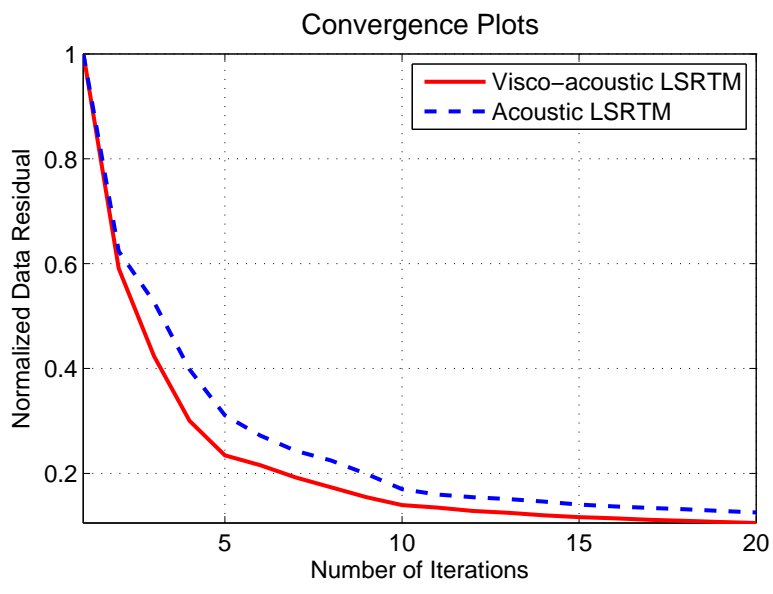

Figure 3: Convergence curves for acoustic and visco-acoustic LSRTM.

\section{ACKNOWLEDGMENTS}

We thank the sponsors of the Center for Subsurface Imaging and Modeling (CSIM) consortium for their support and KAUST Supercomputing Laboratory for providing the computational resources. 
(a) Acoustic RTM Image

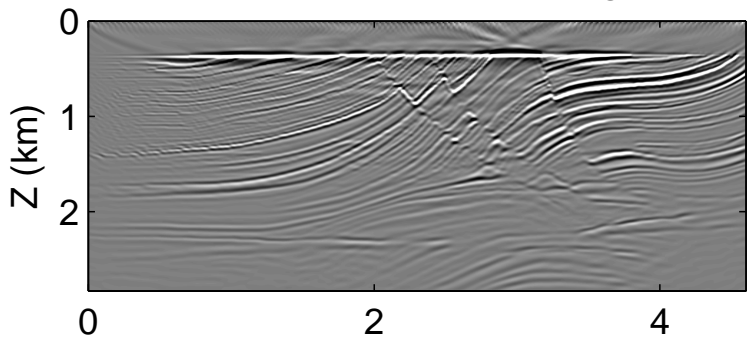

(c) Visco-acoustic RTM Image

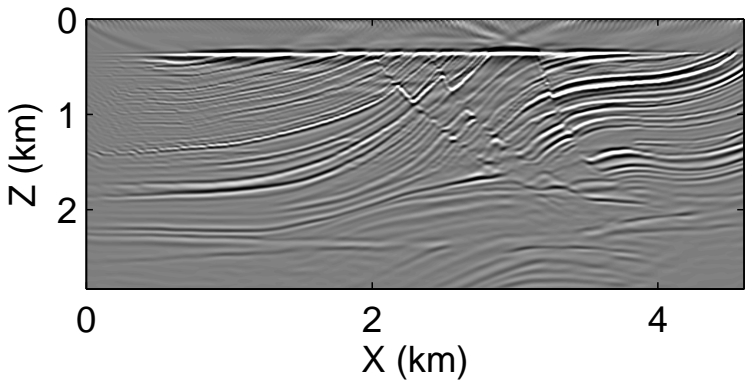

(b) Acoustic LSRTM Image

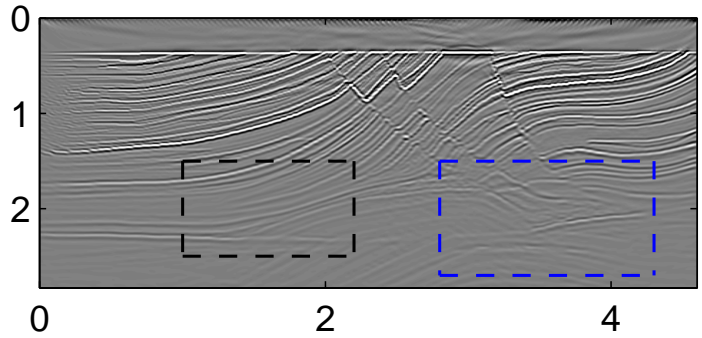

(d) Visco-acoustic LSRTM Image

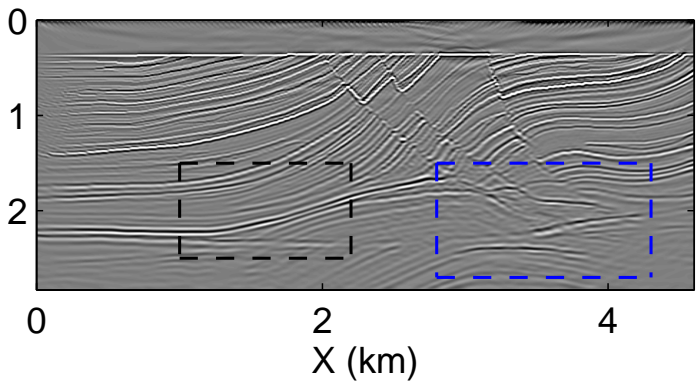

Figure 4: Comparison between images of (a) acoustic RTM, (b) acoustic LSRTM, (c) visco-acoustic RTM, and (d) visco-acoustic LSRTM. All the figures have been plotted in the same scale.

(a) True Reflectivity

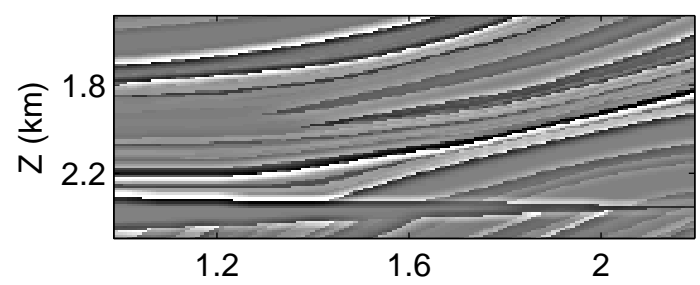

(b) Acoustic LSRTM

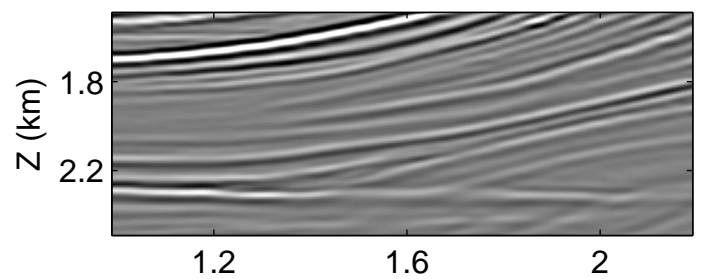

(c) Visco-acoustic LSRTM

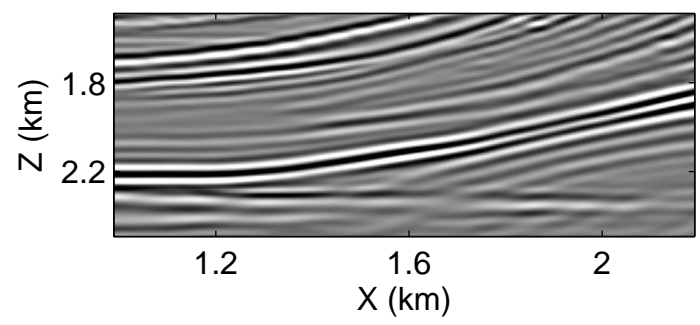

(d) True Reflectivity

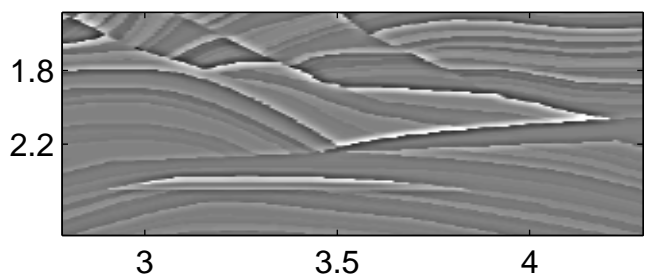

(e) Acoustic LSRTM

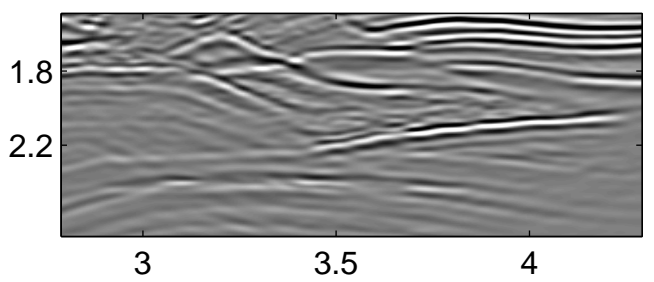

(f) Visco-acoustic LSRTM

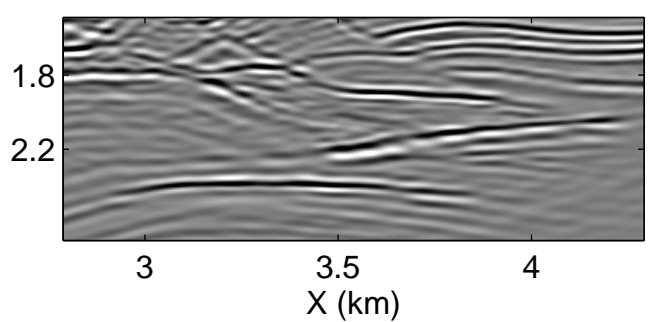

Figure 5: Zoomed view of the black (left) and blue (right) boxes in Figure 4. (a), (d) True reflectivity models used only for comparing the LSRTM images, (b), (e) acoustic LSRTM images, and (c), (f) visco-acoustic LSRTM images. All the figures have been plotted in the same scale. 
http://dx.doi.org/10.1190/segam2013-1131.1

\section{EDITED REFERENCES}

Note: This reference list is a copy-edited version of the reference list submitted by the author. Reference lists for the 2013 SEG Technical Program Expanded Abstracts have been copy edited so that references provided with the online metadata for each paper will achieve a high degree of linking to cited sources that appear on the Web.

\section{REFERENCES}

Aki, K., and P. G. Richards, 1980, Quantitative seismology: Freeman.

Baysal, E., D. D. Kosloff, and J. W. Sherwood, 1983, Reverse time migration: Geophysics, 48, 15141524.

Blanch, J. O., J. O. Robertsson, and W. W. Symes, 1995, Modeling of a constant q: methodology and algorithm for an efficie nt and optimally inexpensive viscoelastic technique: Geophysics, 60, 176184.

Carcione, J. M., D. Kosloff, and R. Kosloff, 1988, Wave propagation simulation in a linear viscoacoustic medium: Geophysical Journal International 93, 393-401.

Christensen, R. M., 1982, Theory of viscoelasticity: An introduction: Academic Press.

Claerbout, J. F., and I. Green, 2008, Basic earth imaging: Citeseer.

Dai, W., P. Fowler, and G. T. Schuster, 2012, Multi-source least-squares reverse time migration: Geophysical Prospecting, 60, 681-695.

Liao, Q., and G. A. McMechan, 1996, Multifrequency viscoacoustic modeling and inversion: Geophysics, 61, 1371-1378.

Liao, Q., and G. A. McMechan, 1997, Tomographic imaging of velocity and Q, with application to crosswell seismic data from the Gypsy pilot site, Oklahoma: Geophysics, 62, 1804-1811.

McMechan, G., 1983, Migration by extrapolation of time-dependent boundary values: Geophysical Prospecting, 31, 413-420.

Nemeth, T., C. Wu, and G. T. Schuster, 1999, Least-squares migration of incomplete reflection data: Geophysics, 64, 208-221.

Robertsson, J. O., J. O. Blanch, and W. W. Symes, 1994, Viscoelastic finite-difference modeling: Geophysics, 59, 1444-1456.

Thorbecke, J. W., and D. Draganov, 2011, Finite-difference modeling experiments for seismic interferometry: Geophysics, 76, no. 6, H1-H18. 